\title{
ON SOME SCHREIER VARIETIES OF UNIVERSAL ALGEBRAS
}

\author{
STEPHEN MESKIN *
}

(Received 30 March 1969)

\section{To Bernhard Hermann Neumann on his 60th birthday}

Communicated by G. E. Wall

\section{Introduction}

Let $\Omega=\left(\Omega_{0}, \Omega_{1}\right)$ where $\Omega_{1}$ is a set of non-empty sets (called operations) and $\Omega_{0}$ is a set of elements (called constants) none of which is a function whose domain belongs to $\Omega_{1}$. An $\Omega$-ALGEBRA is a set $C$ and a function $e$ (the effect) from the disjoint union of $\Omega_{0}$ and $\bigcup_{\omega \in \Omega_{1}} C^{\omega}$ to $C$, where $C^{\omega}$ is the set of all functions from $\omega$ to $C$. Let $P$ be a set of groups $P_{\omega}$ of permutations on $\omega$, one group for each $\omega \in \Omega_{1}$. A $P$ - $\Omega$-ALGEBRA is an $\Omega$-algebra such that $(\rho f) e=(f) e$, for all $\omega \in \Omega_{1}, \rho \in P_{\omega}$ and $f \in C^{\omega}$.

Clearly the collection of all $P$ - $\Omega$-algebras forms a variety. The purpose of this note is to prove the following

Theorem. Subalgebras of free P-Q-algebras are free.

Varieties in which such a theorem holds are called Schreier varieties (see [3] for example). Two special cases are interesting. If $\Omega=(\phi,\{\{\mathbf{1}, 2\}\})$ and $P_{\{1,2\}}$ is the full symmetric group then a $P$ - $\Omega$-algebra is an algebra with a commutative binary operation. If $\Omega$ is arbitrary but $P_{\omega}$ is trivial for all $\omega \in \Omega_{1}$ then every $\Omega$-algebra is a $P$ - $\Omega$-algebra. The validity of the theorem in the latter case especially when all the operations are finite is part of the folklore of universal algebra (see [5], page 136, problem 10 and [2]; note that the proof in [2] is incorrect). The proof is based on the fact that the word problem for these algebras has a very easy solution i.e. two words are equal if and only if they look alike. This idea actually generalizes; all that must be done is to make appropriate definitions. Allowing infinite operations forces one to use transfinite induction rather than ordinary induction but the proof is essentially the same.

In the following argument only the definitions are fully stated. The

* This work was supported in part by NSF Grant GE-7899. 
complete proof of only one statement is included; the remaining proofs are straightforward. For basic definitions see [1] or [4].

I wish to thank Professor S. MacLane, Mr N. Williams and my teacher Professor G. Baumslag for their advice.

\section{The argument}

Let $X$ be a set disjoint from $\Omega_{0}$ no element of which is a function whose domain belongs to $\Omega_{1}$. Then the free $\Omega$-algebra on $X$ is defined by transfinite induction with $C$ equal to the union of $C_{\alpha}$ over all ordinals ${ }^{1} \alpha$, where

$$
\begin{aligned}
C_{0} & =X \cup \Omega_{0} \\
C_{\alpha+1} & =C_{\alpha} \cup\left(\bigcup_{\omega \in \Omega_{1}} C_{\alpha}^{\omega}\right)
\end{aligned}
$$

and for a limit ordinal $\alpha$

$$
C_{\alpha}=\bigcup_{\beta<\alpha} C_{\beta} .
$$

Note that $C$ is the disjoint union of $X, \Omega_{0}$ and the $C^{\omega}$. The effect is the identity map.

The free $P$ - $\Omega$-algebra on $X$ is defined as the quotient of $C$ by a congruence $R$. Define $R$ as the union of $R_{\alpha}$ over all ordinals $\alpha$, where

$$
\begin{aligned}
R_{0} & =\left\{\langle c, c\rangle \mid c \in C_{0}\right\} \\
R_{\alpha+1} & =R_{\alpha} \cup\left\{\langle g, f\rangle \mid g, f \in C_{\alpha}^{\omega} \text { and }\langle(a) \rho g,(a) f\rangle \in R_{\alpha}\right. \\
& \text { for some } \left.\rho \in P_{\omega} \text { and all } a \in \omega\right\}
\end{aligned}
$$

and for a limit ordinal $\alpha$

$$
R_{\alpha}=\bigcup_{\beta<\alpha} R_{\beta}
$$

In the proof that $R$ is a congruence one uses the fact that the $P_{\omega}$ 's are groups - reflexivity and respect for operations because $P_{\omega}$ contains the identity permutation - symmetry from inverses - transitivity from closure. Denote the equivalence class of $c$ by $\bar{c}$ and the induced effect by $e$. Note that if $f$ is a function from $\omega$ to $R$-equivalence classes then $(f) e=\bar{g}$, for some $g$ with $g \in C^{\omega}$ and $(a) f=\overline{(a) g}$ for all $a \in \omega$.

Let $D$ be a collection of $R$-equivalence classes which form a sub-P$\Omega$-algebra, then one can choose as a generating set for $D$

$$
\begin{gathered}
Y=\left\{\bar{c} \in D \mid c \in C^{\omega} \text { and for some } a \in \omega\right. \\
\overline{(a) c} \notin D, \text { or else } c \in X\} .
\end{gathered}
$$

1 Since $\Omega_{1}$ is a set there is a bound on the cardinalities of its elements, hence $C=C_{\alpha}$ for some appropriately large ordinal $\alpha$ and thus $C$ is a set. Functions with different codomains may be identified. 
To show that $Y$ freely generates $D$ we compare $D$ to the free $P$ - $\Omega$-algebra on $Y$ itself. The latter will be the collection of equivalence classes of the set $\Gamma$ built up from $Y$ just as $C$ was built up from $X$. Clearly the identity function on $Y$ extends to a homomorphism $\theta$ of $\Gamma$ onto $D$ which induces a homomorphism $\varphi$ of the collection of equivalence classes onto $D$. Denote the equivalence class of $\gamma \in \Gamma$ by $\overline{\bar{\gamma}}$. The following statements are clear. If $\gamma \in Y$ then $(\overline{\bar{\gamma}}) \varphi=(\gamma) \theta=\gamma$. If $\gamma \in \Omega_{0}$ then $(\overline{\bar{\gamma}}) \varphi=(\gamma) \theta=\bar{\gamma}$. If $\gamma \in \Gamma^{\omega}$ then $(\overline{\bar{\gamma}}) \varphi=(\gamma) \theta=(\gamma \theta) e=\bar{c}$, with $c \in C^{\omega}$ and $(a) \gamma \theta=\overline{(a) c}$ for all $a \in \omega$. In particular if $\gamma \in \Gamma^{\omega}$ then $(\overline{\bar{\gamma}}) \varphi \notin Y$. Indeed if $\gamma$ and $\delta$ are in different sets (from the collection of sets $Y, \Omega_{0}, \Gamma^{\omega}$ for all $\omega \in \Omega_{1}$ ) then $(\overline{\bar{\gamma}}) \varphi \neq(\overline{\bar{\delta}}) \varphi$.

The argument is concluded by proving that $\varphi$ is one-one, i.e. if $\gamma, \delta \in \Gamma$ and $(\overline{\bar{\gamma}}) \varphi=(\overline{\bar{\delta}}) \varphi$ then $\overline{\bar{\gamma}}=\overline{\bar{\delta}}$.

Suppose first of all that $\gamma \in \Gamma_{0}=Y \cup \Omega_{0}$. If $\gamma \in Y$ then $\delta \in Y$. It follows that $\gamma=(\overline{\bar{\gamma}}) \varphi=(\overline{\bar{\delta}}) \varphi=\delta$. If $\gamma \in \Omega_{0}$ then $\delta \in \Omega_{\mathbf{0}}$. In this case $\bar{\gamma}=(\overline{\bar{\gamma}}) \varphi=(\overline{\bar{\delta}}) \varphi=\bar{\delta}$ but these equivalence classes have only one element so $\gamma=\delta$. In either case $\overline{\bar{\gamma}}=\overline{\bar{\delta}}$.

Suppose now that $\gamma \in \Gamma_{x+1}$. Then, by induction, we may assume that $\gamma$ does not belong to $\Gamma_{\alpha}$, hence $\gamma$ and $\delta$ are both in $\Gamma^{\omega}$ for some $\omega$ in $\Omega_{1}$. It follows that $(\overline{\bar{\gamma}}) \varphi=\bar{c}$ and $(\overline{\bar{\delta}}) \varphi=\bar{d}$, where $c$ and $d$ are in $C^{\omega}$ and $\bar{c}=\bar{d}$. By the definition of $R$, it is clear that for some $\rho \in P_{\omega}, \overline{(a) \rho c}=\overline{(a) d}$ for all $a \in \omega$. Consequently $(\overline{\overline{(a) \rho \gamma}}) \varphi=(a) \rho \gamma \theta=\overline{(a) \rho c}=\overline{(a) d}=(a) \delta \theta=(\overline{\overline{(a) \delta)}} \varphi$. By induction, $\overline{\overline{(a) \rho \gamma}}=\overline{\overline{(a) \delta}}$ for all $a \in \omega$; this implies $\overline{\bar{\gamma}}=\overline{\bar{\delta}}$.

Suppose now that $\gamma \in \Gamma_{\alpha}$ where $\alpha$ is a limit ordinal, then $\gamma \in \Gamma_{\beta}$ for some $\beta<\alpha$. Hence by induction, $\overline{\bar{\gamma}}=\overline{\bar{\delta}}$. This concludes the proof of the theorem.

\section{References}

[1] P. M. Cohn, Universal Algebra, (Harper and Row, New York, 1966).

[2] S. Feigelstock, 'A universal subalgebra theorem', Amer. Math. Monthly 72 (1965), 884-888.

[3] J. Lewin, 'On Schreier varieties of linear algebras', Trans. Amer. Math. Soc. 132 (1968), $553-562$.

[4] B. H. Neumann, Universal Algebra, (Notes from New York University, 1962).

[5] R. S. Pierce, Introduction to the Theory of Abstract Algebras, (Holt, Rinehart and Winston, New York, 1968).

The Australian National University Canberra 